\title{
Model-Guided Directional Minimal Path for Fully Automatic Extraction of Coronary Centerlines from Cardiac CTA
}

\author{
Liu Liu ${ }^{1}$, Wenzhe $\mathrm{Shi}^{2}$, Daniel Rueckert ${ }^{2}$, Mingxing $\mathrm{Hu}^{3}$, \\ Sebastien Ourselin ${ }^{3}$, and Xiahai Zhuang ${ }^{4, \star}$ \\ 1 Shanghai Jiaotong Universiy, Shanghai, China \\ 2 Biomedical Image Analysis Group, Imperial College London, UK \\ 3 Centre for Medical Image Computing, University College London, UK \\ 4 Shanghai Advanced Research Institute, Chinese Academy of Sciences, China \\ zhuangxh@sari.ac.cn
}

\begin{abstract}
Extracting centerlines of coronary arteries is a challenging but important task in clinical applications of cardiac CTA. In this paper, we propose a model-guided approach, the directional minimal path, for the centerline extraction. The proposed method is based on the minimal path algorithm and a prior coronary model is used. The model is first registered to the unseen image. Then, the start point and end point for the minimal path algorithm are provided by the model to automate the centerline extraction process. Also, the direction information of the coronary model is used to guide the path tracking of the minimal path procedure. This directional tracking improves the robustness and accuracy of the centerline extraction. Finally, the proposed method can automatically recognize the branches of the extracted coronary artery using the prior information in the model. We validated the proposed method by extracting the three main coronary branches. The mean accuracy of the 56 cases was $1.32 \pm 0.81 \mathrm{~mm}$ and the detection ratio was $88.7 \%$.
\end{abstract}

\section{Introduction}

Cardiac Computer Tomography Angiography (CTA) is widely used in clinical routine for coronary artery studies. Extracting centerlines of the coronary artery is important in the coronary related clinical applications. Since manual extraction and annotation can be time-consuming and skill-demanding, automating this process is becoming increasingly desirable. Many works $[1-4,8]$ focused on extracting the complete tree of the coronary artery. However, it is difficult to automatically recognize and discriminate the branches of the coronary tree without manual interactions. The methods [5-7] based on the minimal path have been used to extract the centerline of a specific vessel or a coronary branch, but the start point and end point, also known as seed points, are commonly manually selected. The detection methods also tend to fail when the seed points are off the

^ Corresponding author.

K. Mori et al. (Eds.): MICCAI 2013, Part I, LNCS 8149, pp. 542-549, 2013.

(C) Springer-Verlag Berlin Heidelberg 2013 


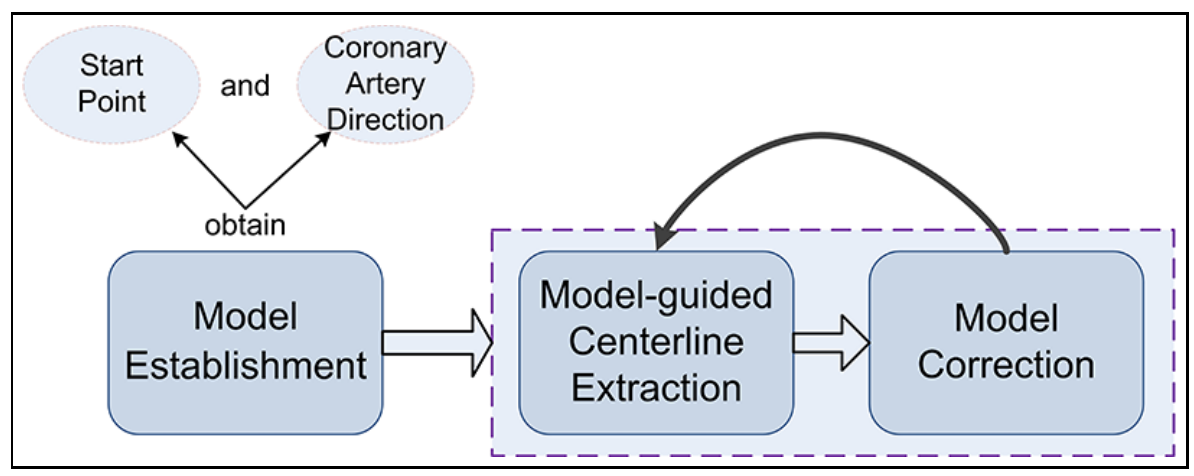

Fig. 1. The flowchart of the proposed directional minimal path

coronary or when the branch has discontinues segments due to bypass surgeries or image artifacts.

In this paper, we propose a new method, the model-guided directional minimal path (DMP), for extracting centerlines of the main branches of the coronary artery. The path tracking in the DMP incorporates the direction information of a prior coronary model, to achieve fast and robust coronary tracking. The start point and the end point required in the minimal path framework are also provided from the prior model. Finally, with the prior information in the model, the branches of the extracted coronary centerlines are automatically recognized, resulting in a fully automated centerline extraction method.

\section{Proposed Method}

The proposed directional minimal path method incorporates a prior model into the framework of the traditional minimal path method [5-7], to extract the centerlines of the coronary artery. The model consists of a cardiac CTA volume, referred to as the CTA model, and the coronary centerlines, referred to as the coronary model. The directional minimal path extraction framework has three key stages: model establishment, model-guided centerline extraction, and model correction, as Fig. 1 shows:

a) In the model establishment, the model is registered to the unseen image, to provide the start point as well as the initial direction for the minimal path.

b) In the model-guided centerline extraction, the start point and the direction from the prior model is incorporated into the framework.

c) The model correction is used to optimize the direction for the model-guided centerline extraction.

The model-guided centerline extraction and model correction are repeated iteratively until the algorithm reaches the neighbor region of the end point of the coronary model or maximal iteration steps. 


\section{$2.1 \quad$ Model Establishment}

In model establishment, the CTA model is first registered to the unseen image using a deformable registration scheme $[11,12]$. The registration consists of three steps, i.e. global affine registration for localization of the whole heart, locally affine registration method for initialization of substructures such as the four chambers and great vessels, and the active control point status free-form deformation registration for refinement of local details. The resultant transformation is then used to map the coronary model onto the image space of the unseen image. The established model provides the start point and the initial direction of the coronary artery for the following directional minimal path procedure. The ideal start point should be the ostia for coronary artery extraction. However, the volumetric registration algorithm tends to produce mismatch of ostia due to its relative small size of volume in the whole heart structure, resulting in less robust coronary extraction. We therefore propose to define the start point to the mean position of the left ostium and the right ostium, for extracting both the left coronary artery and the right coronary artery.

\subsection{Model-Guided Centerline Extraction}

The model-guided centerline extraction is implemented in the framework of the minimal path method, where the cost function of path incorporates the directional information of the coronary model. This is why we call the method the directional minimal path.

In the minimal path framework, the path of the coronary artery is extracted by finding the minimal accumulated cost between the start and end points. The energy function of the path (or curve) $C$ can be define as follows:

$$
E(C)=\int_{\Omega}(P(C(t))+\omega) d t
$$

where $P(\boldsymbol{x})$ denotes the potential or cost at the location $\boldsymbol{x}, \boldsymbol{\omega}$ is a regularization factor, and $t$ is the arch length.

In the directional minimal path, we propose the cost function to include three terms: vesselness [9] $v(\boldsymbol{x})$ based on eigenvalues of the Hessian, similarity [10] $s(\boldsymbol{x})$ based on intensity, and direction $d(\boldsymbol{x})$ based on the prior coronary model. We redefine the cost function $P(\boldsymbol{x})$ as follows:

$$
P(\boldsymbol{x})=\frac{1}{v(\boldsymbol{x})^{\alpha} * s(\boldsymbol{x})^{\beta} * d(\boldsymbol{x})^{\gamma}+\varepsilon} .
$$

where $\varepsilon$ is a small positive value to prevent the singularities, and the parameters $\alpha, \beta$, and $\gamma$, which all set to 1 in our experiments, are used to control the cost contrast. The similarity term $s(\boldsymbol{x})$ can be defined as follows:

$$
s(\boldsymbol{x})=\left\{\begin{array}{l}
e^{-\frac{1}{2}\left(\frac{I(\boldsymbol{x})-\mu_{c a}}{\sigma_{c a}}\right)^{2}}, I<\mu_{c a} \\
1, I \geq \mu_{c a} .
\end{array}\right.
$$




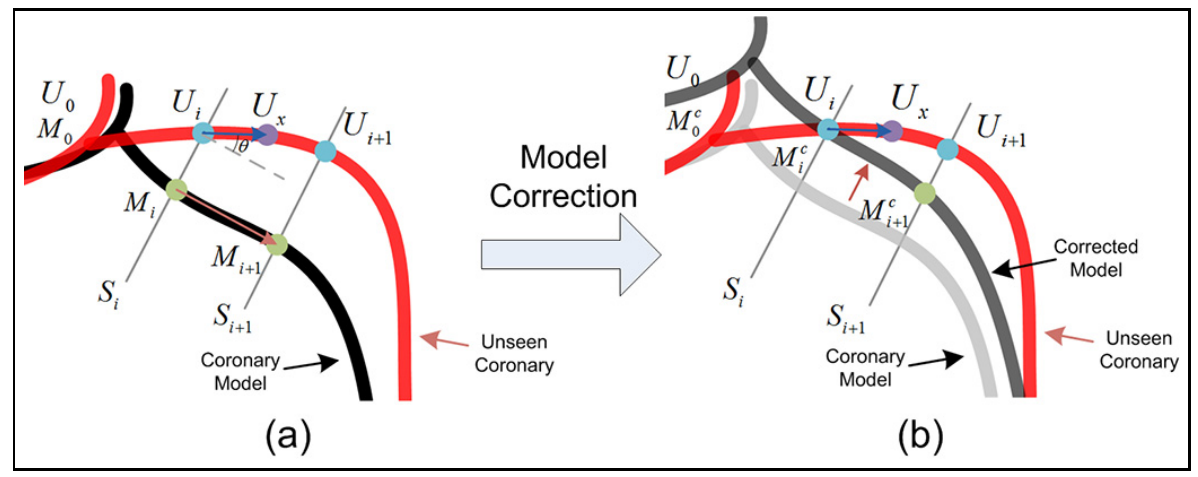

Fig. 2. The model-guided centerline extraction and model correction

where $\mu_{c a}$ and $\sigma_{c a}$ are the intensity mean and standard deviation of the coronary lumen. As the intensity information of the coronary lumen in the unseen image is unknown, we propose to estimate them using the intensity information of the prior model and the intensity information of the contrast enhanced chambers in the unseen image. In the prior model, the mean intensity of the coronary artery, $\mu_{m c a}$, and the enhanced chambers, $\mu_{m e}$, can be accurately computed before the extraction. The contrast enhanced chambers in the unseen image can be identified in the model establishment, where the enhanced chambers in the CTA model were mapped onto the unseen image by the registration. Given the intensity mean of the enhanced chambers of the unseen image, $\mu_{u e}$, is computed, by assuming a constant linear relationship between the intensity value of the contrast enhanced chambers and that of the coronary artery segments, the intensity mean of the coronary artery in the unseen image, $\mu_{u c a}$, is then estimated as follows:

$$
\mu_{c a}=\mu_{u e}+\left(\mu_{m c a}-\mu_{m e}\right) .
$$

In Eq. $(2), d(\boldsymbol{x})$ is determined by the angle $\theta(\boldsymbol{x})$ between the coronary model and the current path in the minimal path method:

$$
\begin{aligned}
& d(\boldsymbol{x})=e^{-\frac{1}{2}\left(\frac{G(\boldsymbol{x})-\mu_{d}}{\sigma_{d}}\right)^{2}}, \\
& G(\boldsymbol{x})=\left\{\begin{array}{l}
\cos (\theta(\boldsymbol{x})), \text { if } \cos (\theta(\boldsymbol{x}))>0 \\
0, \text { else }
\end{array}\right.
\end{aligned}
$$

where $\mu_{d}=1$, and $\sigma_{d}=0.5$ in our experiments.

The angle between the coronary model and the current path is obtained by the piecewise method as illustrated in Fig. 2 (left), where a coronary artery branch of interest is divided into several segments. In a coronary segment, $U_{i}$ and $U_{\boldsymbol{x}}$ denote the start point and the current point in the minimal path process. $M_{i}$ and $M_{i+1}$ denote the start point and the end point of the current segment in the coronary model. The length of each segment $S_{i} S_{i+1}$ can be set as a fixed value. The end point of current segment is regarded as the start point in the 
next segment. Also, to compute the direction information for each candidate path point $\boldsymbol{x}$, one needs to obtain the corresponding segment from the coronary model and employs the direction information of the model for the computation of $\theta(\boldsymbol{x})$. This is achieved by the model correction process.

\subsection{Model Correction}

The prior model gives a good initial estimation after its establishment. However, due to the registration error and the variance of the cardiac anatomy, it is difficult to achieve an accurate match between the coronary model and the corresponding coronary branches in the unseen image. Model correction is embedded with the model-guided centerline extraction to optimize the direction information provided from the model, as follows:

a) For current start point $U_{i}$, search a point on the coronary model, which is the closest point to $U_{i}$. This closest point is referred to as $M_{i}^{c}$.

b) Remap (correct) the coronary model to the unseen image space by aligning $M_{i}^{c}$ to current start point $U_{i}$.

c) Search along the coronary model to find a point $M_{i+1}^{c}$ whose geodesic distance to $M_{i}^{c}$ is the predefined length of a segment.

For each candidate path point $\boldsymbol{x}, \theta(\boldsymbol{x})$ is then defined to the included angle between vector, $\overrightarrow{M_{i}^{c} M_{i+1}^{c}}$, and vector, $\overrightarrow{U_{i} U_{\boldsymbol{x}}}$ as illustrated in Fig. 2 (right). It should be noted that this realignment in model correction does not change the orientation of the coronary model. Instead, it helps to select a better segment from the model to provide more accurate direction for the next iteration, as this selected segment is expected to be more similar to the current coronary segment in the unseen image.

\section{Experimental Results}

We tested the proposed DMP method on eight cardiac CTA volumes, to extract the three main branches of the coronary artery, including the right coronary artery (RCA), left anterior descending artery (LAD), and left circumflex artery (LCX). The centerlines of the coronary branches of interest had been manually annotated in each subject, providing the gold standard for the evaluation.

\subsection{Evaluation Using Synthetic Models}

In this experiment, ten synthetic coronary models are generated for each subject, resulting in 80 cases. The synthetic models were generated by moving the start and end points of the gold standard centerlines, and then deforming the whole coronary. The moving distance was random values between $0-15 \mathrm{~mm}$, and the deformation is computed as follows:

$$
F_{M}(i)=V_{S} \frac{n-1-i}{n-1}+V_{E} \frac{i}{n-1}, i \in(0, n-1) .
$$


Table 1. The results of the two compared methods using synthetic models

\begin{tabular}{lcccc}
\hline \multicolumn{1}{c}{ Methods } & Mean $(\mathrm{mm})$ & Std $(\mathrm{mm})$ & $\operatorname{Max}(\mathrm{mm})$ & Detect Ratio \\
\hline Directional Minimal Path & 0.904 & 0.524 & 2.273 & $97.5 \%$ \\
Vesselness Minimal Path & 1.093 & 0.555 & 2.332 & $50.0 \%$ \\
\hline
\end{tabular}
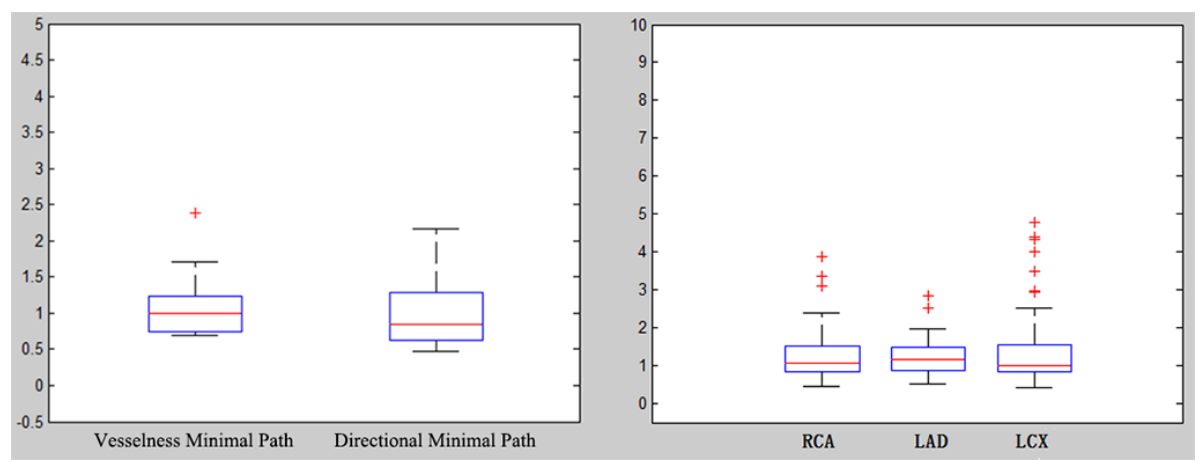

Fig. 3. Box-whisker plots of the centerline extraction results (mm)

where $n$ is the number of points of a gold standard centerline, $i$ is the index of centerline points, $V_{S}$ and $V_{E}$ are the displacements of the start and end points. The deformed coronary was then used as the established coronary model for the DMP algorithm.

The vesselness-based minimal path (VMP) method [7] was also evaluated for comparisons with the proposed DMP method. The start point for the VMP method was manually labeled on the ostia, while the end point was given by the same way as the DMP method. This is because the VMP failed in most of the cases when the start points were the random values as the DMP used. The mean distance between the gold standard and the final results of the successful cases [8] were presented in Table 1, and the box-whisker plots of the two groups of results were given in Fig. 3 (left). Compared with the VMP method, the proposed DMP achieved no worse mean distance on the successful cases, but the detection ratio (success rate of extraction process) was much higher, $97.5 \%$ VS 50\%. It should also be noted that the DMP was fully automatic, while the compared VMP required manual input of the start points.

\subsection{Evaluation Using Real Models}

In this experiment, we employed the leave-one-out strategy, by considering one of the eight subjects as the prior model and the rest as the test dataset, resulting in 56 cases. Fig. 4 provides the visualization result of an extraction result. Table 2 provides the quantitative results and Fig. 3 (right) shows the box-whisker plots of the mean accuracies. The overall extraction accuracy was promising, thought it was slightly worse than the synthetic experiment due to the large difference 
Table 2. The results of directional minimal path method using deformable registration

\begin{tabular}{lcccc}
\hline \multicolumn{1}{c}{ Branches } & Mean $(\mathrm{mm})$ & Std $(\mathrm{mm})$ & $\operatorname{Max}(\mathrm{mm})$ & Detect Ratio \\
\hline Right Coronary Artery & 1.217 & 0.662 & 2.966 & $92.9 \%$ \\
Left Anterior Descending & 1.245 & 0.787 & 3.285 & $82.1 \%$ \\
Left Circumflex Artery & 1.490 & 0.980 & 3.776 & $91.1 \%$ \\
\hline
\end{tabular}

Table 3. The results of directional minimal path method using affine registration

\begin{tabular}{lcccc}
\hline \multicolumn{1}{c}{ Branches } & Mean $(\mathrm{mm})$ & $\mathrm{Std}(\mathrm{mm})$ & $\operatorname{Max}(\mathrm{mm})$ & Detect Ratio \\
\hline Right Coronary Artery & 1.081 & 0.557 & 2.564 & $85.7 \%$ \\
Left Anterior Descending & 1.085 & 0.595 & 2.518 & $83.9 \%$ \\
Left Circumflex Artery & 1.416 & 0.922 & 3.660 & $87.5 \%$ \\
\hline
\end{tabular}

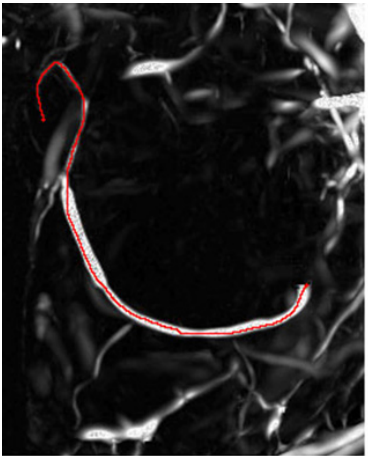

(a)

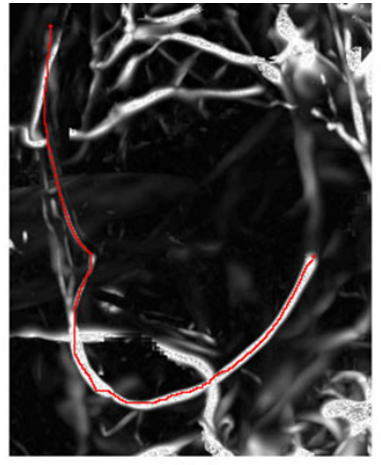

(b)

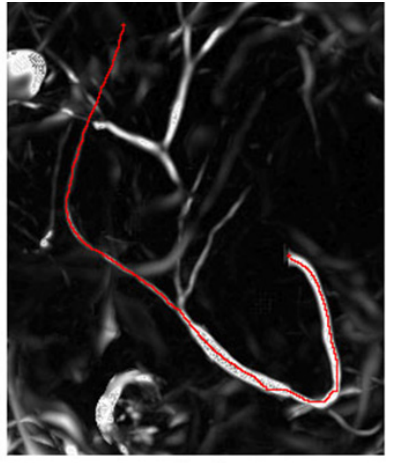

(c)

Fig. 4. The maximal intensity projection of the vesselness with extracted centerlines

of shapes (direction information) between the model and the unseen cases. Also, the maximal errors were generally within the maximal lumen diameters. Finally, the DMP method also demonstrated good robustness against the real cases, as the detection ratios were $92.9 \%, 82.1 \%$, and $91.1 \%$ for the three branches respectively.

Meanwhile, in order to evaluate the effects under the different register methods, the original registration scheme, including affine and nonrigid registration, was replaced by an affine registration method, for the model establishment. The extraction results of this method were presented in Table 3. The detect ratios of the method only using affine registration were worse in RCA and LCA extraction, and marginally better in LAD extraction, compared to the original method which used the deformable registration. However, the accuracy of the centerline extraction of the two methods was not significantly different, as the p-values of them were 0.284 (RCA), 0.095 (LAD), and 0.741 (LCX). 


\section{Conclusions}

In this paper, we presented a novel coronary extraction algorithm, the modelguided directional minimal path (DMP). This approach allows fully automatic extraction of coronary arteries, including the labeling of the main branches. The experiments showed the proposed DMP was robust and accurate in extracting the three main branches. The future work includes extending the DMP for whole coronary tree extraction and validating the algorithm using more clinical datasets.

\section{References}

1. Bouraoui, B., Ronse, C., Baruthio, J., Passat, N., Germain, P.: Fully automatic 3D segmentation of coronary arteries based on mathematical morphology. In: International Symposium on Biomedical Imaging: From Nano to Macro, pp. 1059-1062 (2008)

2. Carrillo, J., Hoyos, M., Davila, E., Orkisz, M.: Recursive tracking of vascular tree axes in 3d medical images. Int. J. Comput. Assist. Radiol. Surg. 1(6), 331-339 (2007)

3. Gülsün, M.A., Tek, H.: Robust vessel tree modeling. In: Metaxas, D., Axel, L., Fichtinger, G., Székely, G. (eds.) MICCAI 2008, Part I. LNCS, vol. 5241, pp. 602611. Springer, Heidelberg (2008)

4. Castro, C., Luengo-Oroz, M., Santos, A., Ledesma-Carbayo, M.: Coronary artery tracking in 3D cardiac CT images using local morpho-logical reconstruction operators. The Midas Journal. In: MICCAI Workshop - Grand Challenge Coronary Artery Tracking (2008)

5. Zhu, N., Chung, A.C.S.: Minimum average-cost path for real time 3D coronary artery segmentation of CT images. In: Fichtinger, G., Martel, A., Peters, T. (eds.) MICCAI 2011, Part III. LNCS, vol. 6893, pp. 436-444. Springer, Heidelberg (2011)

6. Deschamps, T., Cohen, L.D.: Minimal paths in 3D images and application to virtual endoscopy. In: Vernon, D. (ed.) ECCV 2000. LNCS, vol. 1843, pp. 543-557. Springer, Heidelberg (2000)

7. Wink, O., Frangi, A., Verdonck, B., Viergever, M., Niessen, W.: 3D MRA coronary axis determination using a minimum cost path approach. Magn. Reson. Med. 47(6), 1169-1175 (2002)

8. Schaap, M., Metz, C., Walsum, T., et al.: Standardized evaluation methodology and reference database for evaluating coronary artery centerline extraction algorithms. Medical Image Analysis 13(5), 701-714 (2009)

9. Frangi, A.F., Niessen, W.J., Vincken, K.L., Viergever, M.A.: Multiscale vessel enhancement filtering. In: Wells, W.M., Colchester, A.C.F., Delp, S.L. (eds.) MICCAI 1998. LNCS, vol. 1496, pp. 130-137. Springer, Heidelberg (1998)

10. Tang, H., Walsum, T., Onkelen, R., et al.: Semiautomatic carotid lumen segmentation for quantification of lumen geometry in multispectral MRI. Medical Image Analysis 16(6), 1202-1215 (2012)

11. Rueckert, D., Sonoda, L., Hayes, C., Hill, D., Leach, M., Hawkes, D.: Nonrigid registration using free-form deformations: application to breast MR images. IEEE Transactions on Medical Imaging 18(8), 712-721 (1999)

12. Zhuang, X., Rhode, K., Razavi, R., Hawkes, D., Ourselin, S.: A registration-based propagation framework for automatic whole heart segmentation of cardiac MRI. IEEE Transactions on Medical Imaging 29(9), 1612-1625 (2010) 